\title{
Nonlinear Spinor Field in Non-Diagonal Bianchi Type Space-Time
}

\author{
Bijan Saha ${ }^{1, \star}$ \\ ${ }^{1}$ Laboratory of Information Technologies, Joint Institute for Nuclear Research, \\ 141980 Dubna, Moscow region, Russia
}

\begin{abstract}
Within the scope of the non-diagonal Bianchi cosmological models we have studied the role of the spinor field in the evolution of the Universe. In the non-diagonal Bianchi models the spinor field distribution along the main axis is anisotropic and does not vanish in the absence of the spinor field nonlinearity. Hence within these models perfect fluid, dark energy etc. cannot be simulated by the spinor field nonlinearity. The equation for volume scale $V$ in the case of non-diagonal Bianchi models contains a term with first derivative of $V$ explicitly and does not allow exact solution by quadratures. Like the diagonal models the non-diagonal Bianchi space-time becomes locally rotationally symmetric even in the presence of a spinor field. It was found that depending on the sign of the coupling constant the model allows either an open Universe that rapidly grows up or a close Universe that ends in a Big Crunch singularity.
\end{abstract}

\section{Introduction}

Since the first nonlinear generalization of the Dirac equation by Ivanenko [1], the nonlinear Dirac equation (NLD) has emerged as a practical model in many physical systems: (i) extended particles; (ii) the gap solitons in nonlinear optics; (iii) Light solitons in waveguide arrays and experimental realization of an optical analog for relativistic quantum mechanics; (iv) Bose-Einstein condensate in honeycomb optical lattices; (v) Phenomenological models in quantum chromodynamics; (vi) Cosmology; (vii) Chiral models of Skyrme and Faddeev; (vii) Chiral models of Graphene etc. (cf. [2] and references therein).

We study the role of the spinor field in the evolution of the Universe. A key feature of the nonlinear spinor field is that under a suitable choice of the nonlinearity it can (i) eliminate the problem of the initial singularity giving rise to a regular solution; (ii) accelerate the isotropization process of an initially anisotropic space-time and (iii) generate late time acceleration of the expansion of the Universe (cf. [3] and references therein).

Moreover, thanks to its flexibility a nonlinear spinor field can simulate different characteristics of the matter from a perfect fluid to the dark energy and describe the different stages of the evolution of the Universe.

Some recent studies suggest that flexible though it is, the presence of non-diagonal components of the energy-momentum tensor of the spinor field together with Fierz identity impose very severe

\footnotetext{
^e-mail: bijan@jinr.ru
} 
restrictions on the geometry of the Universe as well as on the spinor field, thus justifying our previous claim that the spinor field is very sensitive to the gravitational one. But those studied were performed within the scope of diagonal metrics. The purpose of this report is to extend that study to non-diagonal cases and clarify the role of spinor field in the evolution of the Universe.

\section{Basic equations and their solutions}

We choose the gravitational field in the form

$$
d s^{2}=d t^{2}-a_{1}^{2}(t) d x_{1}^{2}-\left[h^{2}\left(x_{3}\right) a_{1}^{2}(t)+f^{2}\left(x_{3}\right) a_{2}^{2}(t)\right] d x_{2}^{2}-a_{3}^{2} d x_{3}^{2}+2 a_{1}^{2}(t) h\left(x_{3}\right) d x_{1} d x_{2},
$$

where $a_{1}(t), a_{2}(t), a_{3}(t)$ are functions of time and $f\left(x_{3}\right)$ and $h\left(x_{3}\right)$ are some functions of $x_{3}$. Depending on the value of $\delta$ the metric (1) describes Bianchi type-II (BII), Bianchi type-VIII (BVIII) or Bianchi type-IX $(B I X)$ model, respectively:

$$
\delta=-\frac{f^{\prime \prime}}{f}=\left\{\begin{array}{rr}
0, & B I I, \\
-1, & B V I I I, \\
1, & B I X .
\end{array}\right.
$$

The nonlinear spinor field is given by [4]

$$
L_{\mathrm{sp}}=\frac{l}{2}\left[\bar{\psi} \gamma^{\mu} \nabla_{\mu} \psi-\nabla_{\mu} \bar{\psi} \gamma^{\mu} \psi\right]-m_{\mathrm{sp}} \bar{\psi} \psi-F
$$

where $F=F(K)$ is the self-interaction (nonlinear term) with $K$ taking one of the following expressions $\{I, J, I+J, I-J\}$. Here $I=S^{2}=(\bar{\psi} \psi)^{2}$ and $J=P^{2}=\left(i \bar{\psi} \gamma^{5} \psi\right)^{2}$ are invariants constructed from bilinear spinor forms. $\nabla_{\mu}$ is the covariant derivative of the spinor field such that $\nabla_{\nu} \psi=\partial_{\nu} \psi-\Gamma_{\nu} \psi, \quad \nabla_{\nu} \bar{\psi}=$ $\partial_{v} \bar{\psi}+\bar{\psi} \Gamma_{v}$, and $\Gamma_{v}$ is the spinor affine connection. On account of the spinor field equations the energymomentum tensor corresponding to (3) takes the form

$$
\begin{aligned}
T_{\mu}{ }^{\rho} & =\frac{l}{4} g^{\rho v}\left(\bar{\psi} \gamma_{\mu} \partial_{\nu} \psi+\bar{\psi} \gamma_{\nu} \partial_{\mu} \psi-\partial_{\mu} \bar{\psi} \gamma_{\nu} \psi-\partial_{\nu} \bar{\psi} \gamma_{\mu} \psi\right) \\
& -\frac{l}{4} g^{\rho v} \bar{\psi}\left(\gamma_{\mu} \Gamma_{v}+\Gamma_{\nu} \gamma_{\mu}+\gamma_{\nu} \Gamma_{\mu}+\Gamma_{\mu} \gamma_{\nu}\right) \psi-\delta_{\mu}^{\rho}\left(2 K F_{K}-F\right)
\end{aligned}
$$

In order to find the metric functions we have to write the Einstein equations corresponding to (1) and (3) and well as the spinor field equations. We also impose proportionality conditions relating the shear $\sigma$ and expansion $\theta$, that yields

$$
a_{i}=X_{i} V^{Y_{i}}, \quad \prod_{i=1}^{3} X_{i}=1, \quad \sum_{i=1}^{3} Y_{i}=0,
$$

with $V$ being the volume scale defined as $V=a_{1} a_{2} a_{3}$.

From the spinor field equations one obtains the system of equations for the bilinear spinor forms that in our case gives [5]

$$
S=V_{0} / V, \quad V_{0}=\text { const. }
$$

Further, choosing the spinor field nonlinearity in the form

$$
F=\sum_{k} \lambda_{k} I^{n_{k}}=\sum_{k} \lambda_{k} S^{2 n_{k}}=\lambda_{0} S^{2 n_{0}}+\lambda_{1} S^{2 n_{1}}+\lambda_{2} S^{2 n_{2}}
$$

on account of the non-diagonal components from the diagonal Einstein equations and the equations for bilinear spinor forms one finds [5] 


$$
\begin{aligned}
\dot{V} & =Y, \\
\dot{Y} & =\frac{3 \kappa}{2} \sqrt{\frac{q_{2}^{3}}{q_{3}}} \frac{h^{\prime}}{f^{\prime}} V^{3 q_{1} / 2} \Phi_{1}\left(V, A_{0}^{3}, Y\right)+\Phi_{2}\left(V, A_{0}^{3}, Y\right), \\
\dot{A}_{0}^{3} & =\Phi_{1}\left(V, A_{0}^{3}, Y\right),
\end{aligned}
$$

where $A_{0}^{3}=V A^{3}$ with $A^{3}$ being the component of the pseudo-vector $A^{\mu}=\left(\bar{\psi} \gamma^{5} \gamma^{\mu} \psi\right)$ and

$$
\begin{aligned}
\Phi_{1}\left(V, A_{0}^{3}, Y\right)= & -\frac{3 q_{1}}{4 q_{2}} A_{0}^{3} V^{-\left(q_{1}+4 / 3\right)} Y+\frac{b}{\kappa} \sqrt{q_{2}^{5} q_{3}} V^{5 q_{1} / 2-2 / 3}, \\
\Phi_{2}\left(V, A_{0}^{3}, Y\right)= & \frac{1}{4} q_{2}^{4} V^{4 q_{1}+1 / 3}\left(\frac{h^{\prime}}{f}\right)^{2}+\frac{1}{2} q_{2} q_{3} V^{q_{1}+1 / 3} \frac{f^{\prime \prime}}{f} \\
& +\frac{3 \kappa}{2}\left[\left(m_{\mathrm{sp}}+\lambda_{0}\right)+2 \lambda_{1}\left(1-n_{1}\right) V^{1-2 n_{1}}+2 \lambda_{2}\left(1-n_{2}\right) V^{1-2 n_{2}}\right] .
\end{aligned}
$$

Depending on the concrete model we define $f\left(x_{3}\right)$ from (2), whereas the function $h\left(x_{3}\right)$ is defined from

$$
h^{\prime \prime}-\frac{f^{\prime}}{f} h^{\prime}=b f, \quad b=\text { const. }
$$

For the BII model we have $f\left(x_{3}\right)=p x_{3}+q$ and $h\left(x_{3}\right)=c_{1}\left(p x_{3}^{2} / 2+q x_{3}\right)+c_{2}$ for $b=0$ and $h\left(x_{3}\right)=(1 / 3) b p x_{3}^{3}+x_{3}^{2} / 2\left(c_{1} p+b q\right)+c_{1} q x_{3}+c_{2}$ for $b \neq 0$, with $p, q, c_{1}, c_{2}$ being some arbitrary constants.

For the BVIII model we have $f\left(x_{3}\right)=\sinh \left(x_{3}\right)$ and $h\left(x_{3}\right)=\cosh \left(x_{3}\right)$ for $b=0$ and for $b \neq 0$ $h\left(x_{3}\right)=b\left(x_{3} \cosh \left(x_{3}\right)-\sinh \left(x_{3}\right)\right)+c_{1} \cosh \left(x_{3}\right)+c_{2}$, with $c_{1}, c_{2}$ being some arbitrary constants.

For the $B I X$ model we have $f\left(x_{3}\right)=\sin \left(x_{3}\right)$ and $h\left(x_{3}\right)=\cos \left(x_{3}\right)$ for $b=0$ and for $b \neq 0$ $h\left(x_{3}\right)=b\left(\sin \left(x_{3}\right)-x_{3} \cos \left(x_{3}\right)\right)-c_{1} \cos \left(x_{3}\right)+c_{2}$.

In what follows we solve the system (8) numerically. We set $n_{k}=n_{0}: 1-2 n_{0}=0$ which gives $n_{0}=1 / 2$. In this case the corresponding term can be added with the mass term. We assume that $q_{1}>0: 4 q_{1}+1 / 3>0$. For the nonlinear term to be dominant at large time, we set $n_{k}=n_{1}: 1-2 n_{1}>$ $4 q_{1}+1 / 3$, i.e., $n_{1}<1 / 3-2 q_{1}$. And finally, for the nonlinear term to be dominant at the early stage we set $n_{k}=n_{2}: 1-2 n_{2}<0$, i.e., $n_{2}>1 / 2$. Since we are interested in a qualitative picture of the evolution, let us set $\kappa=1, V_{0}=1, q_{2}=1, q_{3}=1$. The initial values are taken as $V(0)=0.01$, $Y(0)=0.1, A_{0}^{3}(0)=1$. We have also set $t \in[0,2]$ with step size 0.001 and $x_{3}=0.2 k \in[0,1]$ with a step $k=0,1, \ldots, 5$.

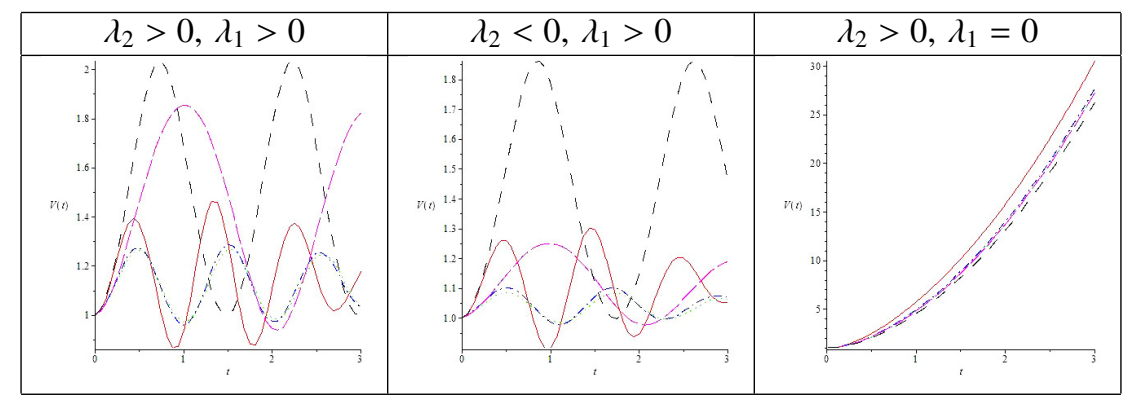

Figure 1. Evolution of $V$ in case $B I X$ space-time with nontrivial $b$ 


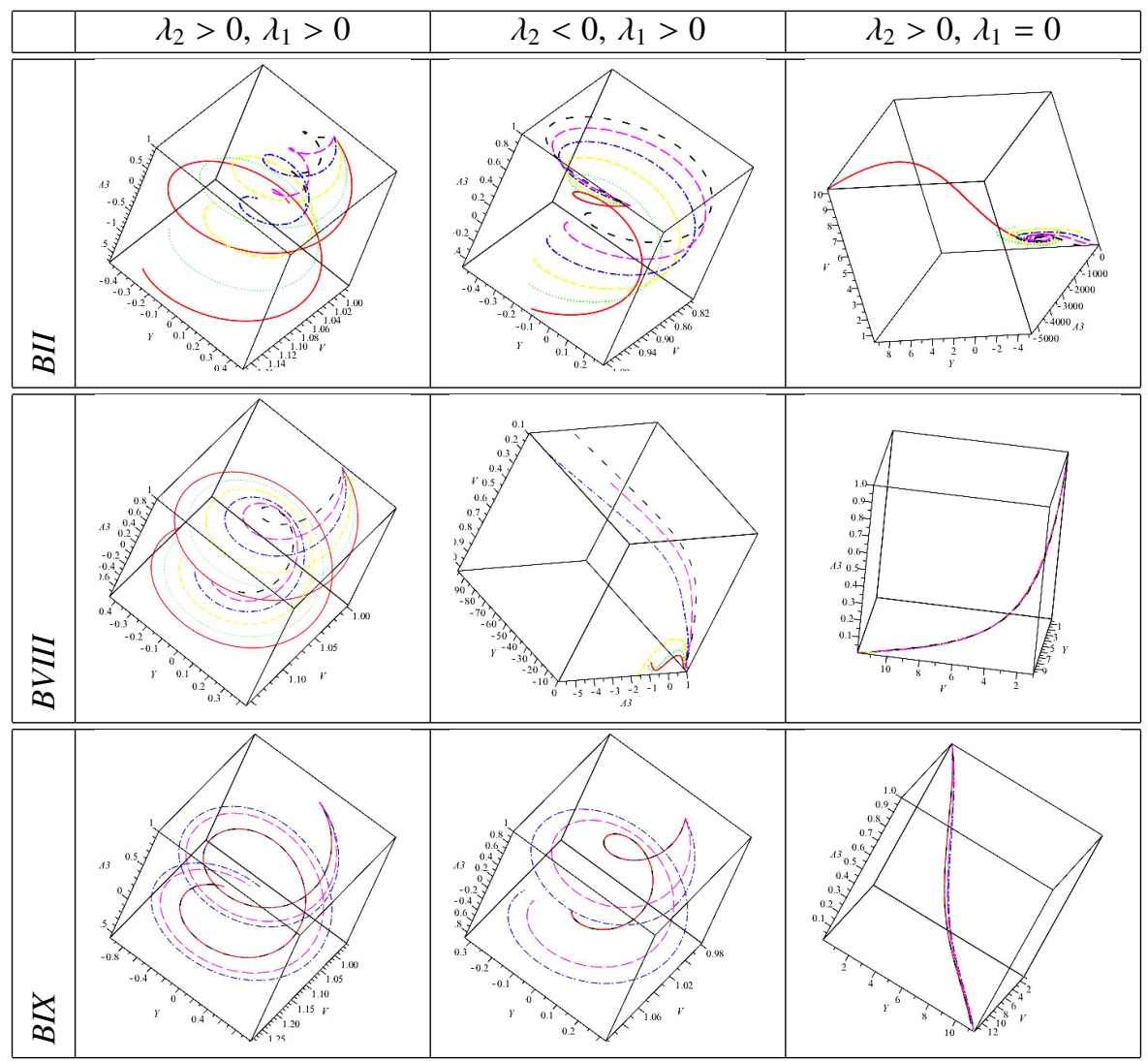

Figure 2. Phase diagram of $\left[V, \dot{V}, A_{0}^{3}\right]$ in case of nontrivial $b$ for $\left[\lambda_{2}>0, \lambda_{1}>0\right],\left[\lambda_{2}<0, \lambda_{1}>0\right]$ and $\left[\lambda_{2}>0, \lambda_{1}=0\right]$ for Bianchi type $-I I, V I I I$ and $I X$

In figure 1 the evolution of $V$ corresponding to these cases is given for $B I X$ model as in the cases we have the qualitatively same picture. In figure 2 we have plotted the phase diagram of $\left[V, \dot{V}, A_{0}^{3}\right]$ for $\left[\lambda_{2}>0, \lambda_{1}>0\right],\left[\lambda_{2}<0, \lambda_{1}>0\right]$ and $\left[\lambda_{2}>0, \lambda_{1}=0\right]$ for Bianchi type $-I I, V I I I$ and $I X$, respectively. In the figures solid, dot, dash, dash-dot,long-dash and space-dash correspond to $k=0,1,2,3,4,5$.

\section{Acknowledgments}

This work is supported in part by a joint Romanian-LIT, JINR, Dubna Research Project, theme no. 05-6-1119-2014/2016.

\section{References}

[1] D. Iwanenko, Physikalische Zeitschrift der Sowjetunion 13, 141-150 (1938)

[2] B. Saha, Eur. Phys. J. Plus 131, 242-17 (2016)

[3] B. Saha, Astrophys. Space Sci. 331, 243 (2011)

[4] B. Saha, Phys. Rev. D 64, 123501 (2001)

[5] B. Saha, ArXiV. 1705.07773 [gr-qc] (2017) 